\title{
Vinorelbine neurotoxicity: clinical and neurophysiological findings in 23 patients
}

\author{
A Pace, L Bove, C Nisticò, M Ranuzzi, P Innocenti, A Pietrangeli, E Terzoli, B Jandolo
}

\begin{abstract}
Vinorelbine ( $5^{\prime}$-noranhydrovinblastine) is a new semisynthetic antineoplastic vinca alkaloid which interferes with axonal transport, inducing spiralisation of axonal microtubules and resulting in peripheral neurotoxicity. A prospective detailed neurological and electrophysiological evaluation was performed in 23 patients treated with $25 \mathrm{mg}$ vinorelbine a week. All patients developed a sensorymotor distal symmetric axonal neuropathy. The neurotoxicity increased with cumulative vinorelbine doses and peripheral neuropathy was mild or moderate in most patients. After discontinuation of vinorelbine treatment, neuropathic signs and symptoms were partially reversible.
\end{abstract}

$(\mathcal{F}$ Neurol Neurosurg Psychiatry 1996;61:409-411)

Keywords: vinorelbine; neurotoxicity; neuroprotection

Vinorelbine (5'-noranhydrovinblastine) is a new semisynthetic vinca alkaloid with established activity in advanced breast cancer and non-small cell lung carcinoma, both as a single agent and in combination with other drugs. ${ }^{1}$ The antitumour activity of this cell cycle dependent agent seems to be related to the inhibition of tubulin polymerisation into microtubules ${ }^{2}$ and cell blockage in metaphase.

The well known peripheral neurotoxicity of vinca alkaloids is due to the biochemical similarities in structure between the filaments of the mitotic spindle and the neurotubules and filaments of the axon. In the same manner as other vinca alkaloids vinorelbine interferes with axonal transport, inducing spiralisation of axonal microtubules, but this action was noted at a higher concentration than with vincristine and vinblastine. ${ }^{3}$ For this reason vinorelbine seems to cause less neurotoxicity than other vinca alkaloids.

Clinical trials reported a very mild neurotoxicity with distal paraesthesiae and decrease or abolition of tendon jerks, ${ }^{2}$ but studies describing the clinical and neurophysiological profile of this neuropathy are not available in the medical literature.

We performed a prospective detailed neurological and electrophysiological evaluation in
23 patients treated with vinorelbine with the aim of defining the incidence, severity, neurophysiological profile, and reversibility of vinorelbine neurotoxicity.

\section{Materials and methods}

From April to November 1994, 23 patients affected by advanced breast carcinoma and treated with vinorelbine were enrolled. Patients were eligible if not pretreated with other neurotoxic drugs. Patients presenting with other risk factors for neuropathies, such as diabetes mellitus, alcoholism, and paraneoplastic or dismetabolic syndromes were not included. We adopted an unconventional intensified schedule of vinorelbine $(25 \mathrm{mg}$ a week given intravenously sequentially for 24 weeks, plus granulocyte colony stimulating factor). Five interrupted the treatment after the 12 th administration of vinorelbine because of progression of disease.

Patients were aged between 32 and 70 years and had measurable disease, performance status between $0-1$ on the Eastern Cooperative Oncology Group criteria ${ }^{4}$ in 19 patients, between $2-3$ in four, normal bone marrow, hepatic, and renal function, and a life expectancy of a least 12 weeks. They gave informed consent.

Neurological evaluation and neurophysiological examination were performed before and after 4, 12 , and 24 cycles of therapy and (in six patients) six months after the end of therapy. Clinical neurological examination, performed independently by two neurologists (AP or LB), included a standardised history for detection of neuropathic symptoms and a complete evaluation of pinprick and vibratory sensation, strength, and deep tendon reflexes. Neuropathic signs and symptoms were scored using a questionnaire designed for detection of sensory disturbances (paraesthesia, pain, burning in feet or fingers) and symptoms of muscle weakness (upper arm, hand, thigh, and leg weakness) experienced by patients. We adopted a modified version of the neurological symptom score (NSS) by Dyck et al. ${ }^{5}$ grading the severity of symptoms as mild 1 , moderate 2 , and severe $>2$.

Nerve conduction was measured in the median, peroneal, and sural nerves with surface electrodes at constant skin temperature 
Table 1 Neurotoxicity score based on clinical subjective symptoms, clinical signs, and neurophysiological findings

\begin{tabular}{|c|c|c|c|c|}
\hline & \multicolumn{4}{|c|}{ Neurotoxicity score } \\
\hline & 0 & 1 & 2 & 3 \\
\hline $\begin{array}{l}\text { NSS } \\
\text { Vibratory sensation }\end{array}$ & $\stackrel{0}{\text { Normal }}$ & $\begin{array}{l}1 \\
\text { Slightly } \\
\text { reduced }\end{array}$ & $\begin{array}{l}2 \\
\text { Reduced }\end{array}$ & $\begin{array}{l}>2 \\
\text { Absent }\end{array}$ \\
\hline $\begin{array}{l}\text { Tendon reflexes } \\
\text { Median sensory amplitude }\end{array}$ & $\begin{array}{l}\text { Normal } \\
\text { Normal }\end{array}$ & $\begin{array}{l}\text { AJ reduced } \\
10-25 \%\end{array}$ & $\begin{array}{l}\text { AJ absent } \\
25-50 \%\end{array}$ & $\begin{array}{l}\text { All absent } \\
>50 \% \\
\text { reduction }\end{array}$ \\
\hline Peroneal amplitude & Normal & $10-25 \%$ & $25-50 \%$ & $\begin{array}{l}>50 \% \\
\text { reduction }\end{array}$ \\
\hline Sural amplitude & Normal & $10-25 \%$ & $25-50 \%$ & $\begin{array}{l}>50 \% \\
\text { reduction }\end{array}$ \\
\hline
\end{tabular}

Mild neurotoxicity $=$ score $<4$; moderate neurotoxicity $=$ score $5-10$; severe neurotoxicity $=$ score > 10; modified from Chandhry et al.

NSS = Neurological symptom score; $\mathrm{AJ}=$ ankle jerk.

$\left(34^{\circ} \mathrm{C}\right.$ at least). Action potential amplitudes were measured from peak to peak for the sensory response (SAP) and using the negative phase amplitudes for the motor response (MAP). Values were considered abnormal if they exceeded the normal range of our laboratory. A measurement of sympathetic skin response (surface electrode stimulation at the wrist and contralateral registration with active electrode on the palm) were also made. ${ }^{6}$

The pretreatment and post-treatment values for all the variables were compared by analysis of variance (ANOVA) for repeated measures. A cumulative neurotoxicity score was calculated for each patient by scoring the severity of symptoms and clinical and electrophysiological signs using a modified version of the neuropathy score of Chaudry et al. (table 1). ${ }^{7}$ The severity of neurotoxicity was graded on the basis of the obtained score as mild neurotoxicity $=$ total score 1 to 4 ; moderate neurotoxicity $=$ score $5-10$; severe neurotoxicity $=$ score $>10$.

\section{Results}

\section{NEUROLOGICAL EVALUATION}

The pretreatment evaluation was normal in all the patients; after four cycles of vinorelbine 11 out of 23 patients $(47 \%)$ showed clinical symptoms and signs of mild neuropathy including reduced or absent ankle jerks (11 patients) and distal paraesthesia prevailing in the lower limbs (three patients (13\%)).

After 12 cycles of therapy 11 out of 23 patients $(47 \%)$ complained of dysaesthesia in the hands and feet; neurological examination showed deep tendon hypoareflexia in 17 patients (73\%) and perimalleolar hypopallaesthesia in two $(8 \cdot 6 \%)$.

After 24 cycles the clinical evaluation performed in 18 out of 23 patients disclosed disaesthesia in the lower limbs in nine patients

Table 2 Mean (SD) of peroneal MAPs $(m V)$ and median and sural SAPs $(\mu V)$ before and after 4, 12 and 24 courses of therapy

\begin{tabular}{|c|c|c|c|c|c|c|}
\hline & \multirow[b]{2}{*}{ Normal } & \multirow[b]{2}{*}{ Baseline } & \multicolumn{4}{|l|}{ Courses } \\
\hline & & & $4(n=23)$ & $12(n=23)$ & $24(n=18)$ & Pvalue \\
\hline $\begin{array}{l}\text { Median } \\
\text { Peroneal } \\
\text { Sural }\end{array}$ & $\begin{array}{r}21 \cdot 2(4 \cdot 6) \\
5 \cdot 2(2 \cdot 2) \\
16 \cdot 8(6 \cdot 3)\end{array}$ & $\begin{array}{c}25 \cdot 2(11 \cdot 6) \\
5 \cdot 6(1 \cdot 8) \\
10.9(6)\end{array}$ & $\begin{array}{r}23.7(9 \cdot 1) \\
4.4(1.9) \\
9.2(5.4)\end{array}$ & $\begin{array}{r}16.4(7.5) \\
3.7(1.7) \\
8.7(5.5)\end{array}$ & $\begin{array}{c}16.4(7) \\
3.0(1.4) \\
8.5(9.3)\end{array}$ & $\begin{array}{l}0.002 \\
0.001 \\
0.05\end{array}$ \\
\hline
\end{tabular}

$P$ value refers to amplitudes after 24 courses $v$ baseline.
$(50 \%)$, absence of deep tendon reflexes in 10 (55\%), and hypoareflexia of ankle jerk in seven $(39 \%)$. Only one patient was free of neuropathic signs or symptoms.

In six patients long term neurological evaluation was performed six months after the end of treatment with vinorelbine. Clinical examination showed absence of subjective symptoms, partial (in one patient) or complete recovery of deep tendon reflexes, and normal pinprick and deep sensation in all patients.

\section{NEUROPHYSIOLOGICAL STUDIES}

The pretreatment evaluation showed normal neurophysiological values in all patients except a reduced amplitude of the SAP of the sural nerve in three patients and of the median nerve in one patient.

After four courses of vinorelbine the mean values of nerve conduction velocities, distal latencies, MAPs and SAPs, and sympathetic skin response were normal. A reduction by more than $50 \%$ of baseline values was detected in peroneal amplitude in six patients, in sural amplitude in four, and in median sensory amplitude in one (table 2).

After 12 courses of treatment the mean peroneal MAP amplitude was significantly reduced by $36 \%$ of baseline values $(\mathrm{P}<0.001)$; the mean median SAP amplitude was significantly reduced by $29 \%(P<0.001)$; the mean sural SAP amplitude was reduced by $22 \%(P<0.05)$. Conduction velocity, distal latency, and sympathetic skin response values remained unchanged. Peroneal MAP amplitude was decreased by more than $50 \%$ in seven patients, sural SAP in four, and median SAP in one.

At the end of treatment after 24 cycles of vinorelbine the mean values of peroneal amplitude obtained in 18 out of 23 patients were decreased by $47 \%$ compared with pretreatment values $(P<0.001)$, mean median SAP amplitude decreased by $38 \%(\mathrm{p}<0.005)$, and sural SAP decreased by $26 \% \quad(P<0.05)$. Conduction velocities, distal latencies, and sympathetic skin response were still normal. In nine patients peroneal MAP amplitude fell below $50 \%$ of baseline values, sural SAP in seven (absent response in one) and median SAP in five.

Six months after vinorelbine discontinuation electrophysiological data showed in six patients an improvement of median and sural SAP (one patient was absent at examination after 24 cycles) and partial recovery of peroneal MAP amplitudes.

\section{NEUROPATHIC SCORE}

After four cycles of vinorelbine 20 out of 23 patients $(87 \%)$ showed symptoms and signs of neurotoxicity: in 16 patients $(69 \%)$ the neurophatic score was < five (mild neurotoxicity), in four $(17 \%)<10$ (moderate neurotoxicity). After 12 cycles nine (39\%) patients complained of mild signs and symptoms and scored $<5,14(60 \%)$ had moderate neurotoxicity, scores $<10$. After 24 cycles of chemotherapy 12 patients $(66.6 \%)$ showed moderate neurotoxicity with a score of 5 to 10 
and $\operatorname{six}(33.3 \%)$ severe neurotoxicity with a score $>10$. However, in no patient did the severity of neurotoxicity require discontinuation of treatment, and no patients had a World Health Organisation (WHO $)^{8}$ disability score $>2$.

Total neurotoxicity score was significantly related to the number of cycles of vinorelbine $(r=0.9)$.

\section{Discussion}

Peripheral neurotoxicity due to vinorelbine treatment was described as occurring less often than after treatment with other vinca alkaloids. The reported incidence in phase II oncological studies varied from $6 \%$ to $29 \% .{ }^{9}$

The WHO neurotoxicity grading system commonly used in oncological series, however, does not permit the real incidence and the neuropathy profile to be evaluated.

The detailed neurological and neurophysiological evaluations and the neurotoxic score system used in our study are sensitive methods for detecting early peripheral neurotoxicity and allowed a precise neuropathy assessment.

Our data show that all the patients treated with vinorelbine developed a mild distal sensory-motor, symmetric neuropathy of axonal type. Most patients had reduced or abolished deep tendon reflexes and sensory symptoms with distal paraesthesiae, hypoaesthesia, and hypopallaesthesia, showing an involvement of both small and large calibre sensory fibres; electrophysiological findings confirm the involvement of both motor and sensory fibres with decreased amplitude of MAPs and SAPs and normal conduction velocities. These findings are indicative of a distal axonopathy similar to that induced bypiridoxine. ${ }^{10}$

Follow up data after six months confirmed that this kind of neuropathy may reverse, like that with distal axonal neuropathies due to vincristine and paclitaxel.

The degree of clinical and neurophysiological impairment correlates with the number of courses given $(r=-0.9)$ and shows that this neuropathy is dose-dependent. At the dosage used in our study vinorelbine induced slight neuropathic signs and symptoms but did not seem to be dose limiting.

The sympathetic skin response, a simple method of assessing dysfunction of unmyeli- nated axons in axonal neuropathy proposed by Shahani et $a l,{ }^{6}$ did not show involvement of small unmyelinated $\mathrm{C}$ fibres.

The very high incidence of vinorelbine neurotoxicity in our study could be explained by the detailed clinical and electrophysiological methods used and, perhaps, with the high dose intensity of the schedule adopted. Although the severity of vinorelbine neuropathy does not seem clinically relevant, the use of this drug in combination with other neurotoxic agents such as paclitaxel or platinum compounds, may aggravate the neurotoxic damage. Some studies ${ }^{1112}$ suggest that administration of several neuronotrophic factors (nerve growth factor, ACTH analogues, and others) can prevent or limit neurotoxicity of many drugs. We think that to define the real incidence and the precise pathological profile of neurotoxicity from antineoplastic drugs, using standardised methods, is extremely important both for evaluating the drug itself, and in evaluating the potential benefit of neuroprotective agents.

The study was supported in part by Italian National Research Council (CNR) grant 9400985CT04. We are grateful to Professor M Manfredi for his critical review and to Dr D Giannarelli for her statistical suggestions.

1 Zhou XJ, Rahmani R. Preclinical and clinical pharmacology of vinca alkaloids. Drugs 1992:44(suppl):1-16.

2 Gasparini G, Caffo O, Barni S, et al. Vinorelbine is an active antiproliferative agent in pretreated advanced breast cancer patients: a phase II study. $\mathcal{F}$ Clin Oncol 1994;12:2094-101.

3 Honeker JA. A summary of vinorelbine (Navelbine) safety data from North American clinical trials. Semin Oncol 1994;21 (suppl 10):42-7.

4 Oken MM. Toxicity and response criteria of the Eastern Cooperative Oncology Group. Am $\mathcal{F}$ Clin Oncol 1982;5:

5 Dyck PJ, Sherman WR, Hallcher LM, Service FG, O'Brien PC, Grina LA, et al. Human diabetic endoneurial sorbitol, fructose and myo-inositol related to sural nerve morphometry. Ann Neurol 1980;8:590-6.

6 Shahani BT, Halperin J, Boulu P, Cohen J. Sympathetic skin response-a method of assessing unmyelinated axon skin response-a method of assessing unmyelinated axon
dysfunction in peripheral neuropathies. $\mathcal{f}$ Neurol
Neurosurg Psychiatry 1984;47:536-42.

7 Chaudry V, Rowinsky EK, Sartorius SE, Donehower RC, Cornblath DR. Peripheral neuropathy from Taxol and Cisplatin combination chemotherapy: clinical and electrophysiological studies. Ann Neurol 1994;35:304-11.

8 World Health Organisation. WHO Handbook for reporting results of cancer treatment. Geneva: WHO, 1979:N48.

9 Cvitkovic E, Izzo J. The current and future place of vinorelbine in cancer therapy. Drugs 1992;44(suppl 4):36-45.

10 Schaumburg HH, Spencer PS. Toxic neuropathies. Neurology 1979;29:429-31.

11 Windebank AJ, Gordin Smith A, Russel JW. The effect of nerve growth factor, ciliary neurotrophic factor, and ACTH analogs on cisplatin neurotoxicity in vitro. Neurology 1994;44:488-94.

12 Apfel SC, Lipton RB, Arezzo JC, Kessler JA. Nerve growth factor prevents toxic neuropathy in mice. Ann Neurol 1991;29:87-90. 